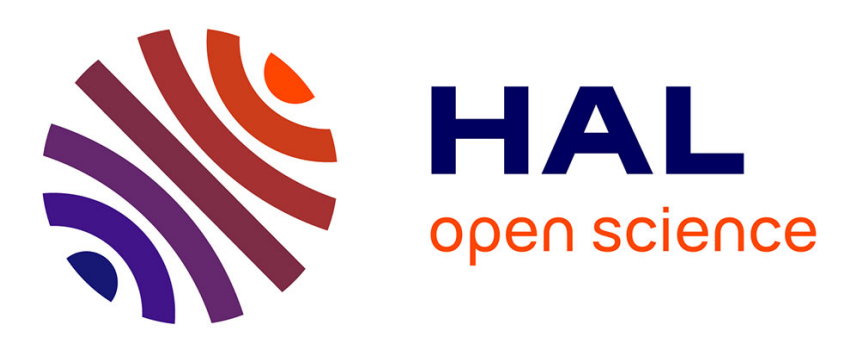

\title{
Polymères nématogènes : seuil d'apparition de la texture à fils fins
}

B. Millaud, A. Thierry, A. Skoulios

\section{To cite this version:}

B. Millaud, A. Thierry, A. Skoulios. Polymères nématogènes: seuil d'apparition de la texture à fils fins. Journal de Physique Lettres, 1979, 40 (22), pp.607-608. 10.1051/jphyslet:019790040022060700 . jpa-00231698

\section{HAL Id: jpa-00231698 https://hal.science/jpa-00231698}

Submitted on 1 Jan 1979

HAL is a multi-disciplinary open access archive for the deposit and dissemination of scientific research documents, whether they are published or not. The documents may come from teaching and research institutions in France or abroad, or from public or private research centers.
L'archive ouverte pluridisciplinaire HAL, est destinée au dépôt et à la diffusion de documents scientifiques de niveau recherche, publiés ou non, émanant des établissements d'enseignement et de recherche français ou étrangers, des laboratoires publics ou privés. 


\title{
Polymères nématogènes : seuil d'apparition de la texture à fils fins
}

\author{
B. Millaud, A. Thierry et A. Skoulios \\ Centre de Recherches sur les Macromolécules, C.N.R.S., 6, rue Boussingault, 67083 Strasbourg Cedex, France
}

(Reçu le 23 juillet 1979, révisé le 1er octobre 1979, accepté le 1er octobre 1979)

\begin{abstract}
Résumé. - Nous rapportons ici quelques observations de textures de phases nématiques lyotropes de poly(azométhines) dans l'acide sulfurique. Nous avons mis en évidence l'existence de deux textures différentes : l'une à fils fins, l'autre mal définie. Le seuil d'apparition de la texture à fils fins a été relié à l'existence d'une concentration minimale en extrémités de chaînes.
\end{abstract}

\begin{abstract}
Some observations of the textures of the lyotropic nematic phase of poly(azomethines) in sulphuric acid are reported. The effects of varying concentration, temperature and molecular weight have been studied and the existence of two distinct textures has been found : one threaded and the other ill-defined. We suggest that the threaded texture can only occur when there is a sufficient concentration of chain ends.
\end{abstract}

Dans un article récent [1], nous avons étudié la texture à fils fins des mésophases nématiques lyotropes de certains polymères à chaînes plus ou moins rigides. Les systèmes considérés étaient des solutions concentrées dans l'acide sulfurique pur de polyamides aromatiques et d'une polyazométhine. Les observations faites au microscope polarisant étaient interprétées, en ce qui concerne l'arrangement moléculaire autour des lignes de disinclinaison, à l'aide d'un modèle de divergence radiale pure.

Plus récemment [2], nous avons été amenés à compléter nos expériences par l'examen d'un certain nombre d'autres polymères nématogènes. Parmi ceux-ci figure en particulier la poly(téréphtal, méthylp-phénylènediimine) dont la formule s'écrit :<smiles>CNc1ccc(N=Cc2ccc(C)cc2)c(C)c1</smiles>

Ce polymère était connu pour donner des phases nématiques thermotropes [3]. Nous avons constaté qu'en solution dans l'acide sulfurique pur, il fournit également des mésophases lyotropes.

Nous disposions d'une large variété d'échantillons de ce polymère de masse moléculaire s'échelonnant entre 1500 et 7000 . Ainsi, nous avons pu réaliser une étude assez systématique des conditions d'apparition de la phase nématique en fonction de la masse moléculaire, de la température et de la concentration des solutions. Dans la figure 1, nous avons résumé
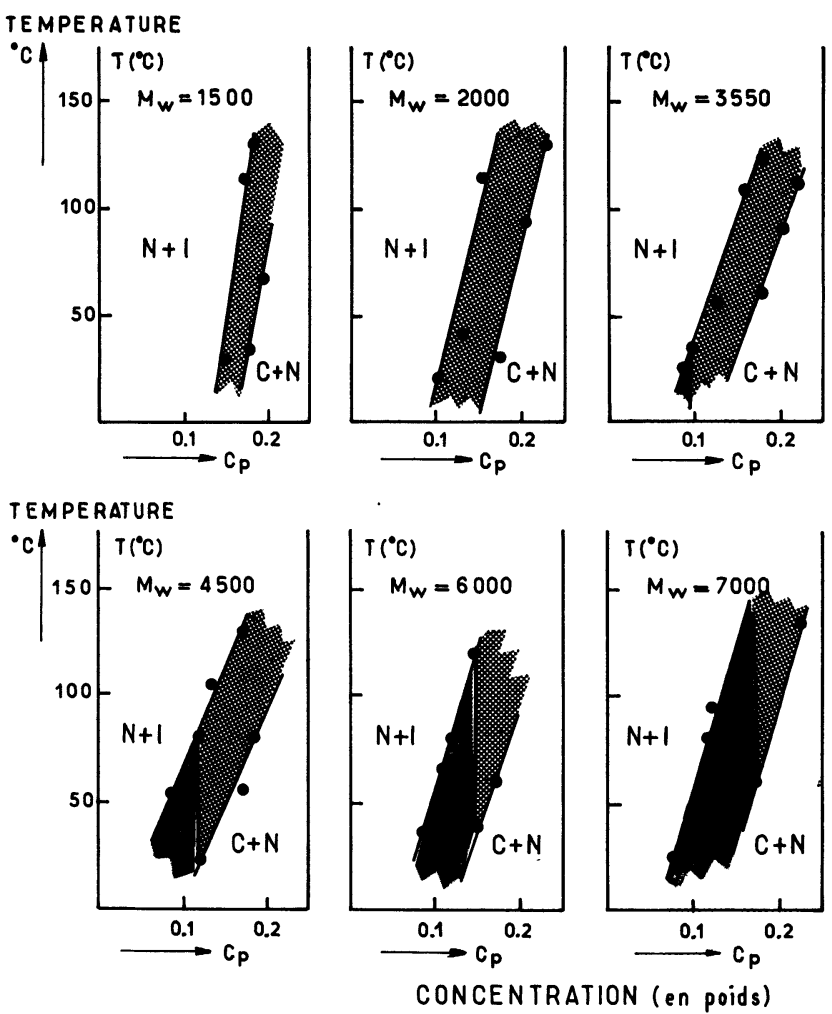

Fig. 1. - Domaine d'existence de la phase nématique du Me-PNPM dans $\mathrm{H}_{2} \mathrm{SO}_{4}$ pour diverses masses moléculaires. : zone nématique à fils fins; mal définie.

[Region of existence of the nematic phase of Me-PNPM in $\mathrm{H}_{2} \mathrm{SO}_{4}$ for different molecular weights. : threaded texture of the nematic phase; : ill-defined texture of the nematic phase.] 
l'essentiel de nos observations, en n'y représentant que le domaine d'existence de la phase nématique, limité de part et d'autre, par les zones de démixtion avec la solution isotrope et le cristal respectivement.

Un examen détaillé des textures présentées par les phases nématiques nous a permis de constater qu'elles sont de deux types, soit une texture à fils fins, bien développée, identique à celle observée précédemment (voir fig. 2b, réf. [1]), soit alors une texture mal définie, comportant des bandes plus ou moins sombres, floues et diffuses (texture analogue à celle montrée dans la figure $2 c$, réf. [1]). Rappelons qu'en accord avec nos observations antérieures [1], la texture à fils n'apparaît qu'après relaxation des distorsions créées dans l'échantillon par l'application de légères contraintes mécaniques; la durée de cette relaxation varie avec les conditions expérimentales (viscosité, masse moléculaire, concentration, ...). La distinction entre la texture à fils et la texture diffuse est aisée : en règle générale, la première apparaît très rapidement, après une relaxation de moins d'une minute, alors que la seconde n'évolue pas et ne relaxe pas pendant toute la durée de nos observations, qui n'était d'ailleurs jamais inférieure à quinze minutes.

La texture à fils apparaît systématiquement et dans tout le domaine d'existence de la phase nématique, lorsque la masse moléculaire est suffisamment faible; pour les masses élevées, elle n'existe que si les solutions sont suffisamment concentrées (voir zones hachurées, fig. 1). Ce changement de comportement est net et bien défini. Pour un échantillon de polymère de masse moléculaire donnée, il est possible expérimentalement de modifier le type de texture observée, en faisant simplement varier la concentration et en passant alternativement de part et d'autre d'un seuil de concentration que nous estimons pouvoir définir à $1-2 \%$ près. Si la position de ce seuil ne semble pas affectée par la température, elle varie au contraire, très rapidement avec la masse moléculaire.

En réalité, aux erreurs de l'expérience près, il semble bien que cette concentration limite soit directement proportionnelle à la masse moléculaire, les points représentant l'évolution de la concentration pondérale $C_{\mathrm{p}}$ en fonction de la masse moléculaire moyenne en poids $M_{\mathrm{w}}$ se situant sur une droite passant par l'origine (fig. 2). Ceci suggère que, lors de l'apparition de la texture à fils, la concentration molaire des solutions, ou, ce qui revient au même, la densité d'extrémités de chaînes dans le milieu, est à peu près constante. Pour démontrer cette constance, nous aurions dû, en toute rigueur, porter dans le graphique, non pas $C_{\mathrm{p}}$ en fonction de $M_{\mathrm{w}}$, mais plutôt la concentration volumique $C_{\mathrm{v}}$ (masse de polymère par unité de volume) en fonction de la masse moléculaire moyenne en nombre $\left(M_{\mathrm{n}}\right)$. Cette représentation

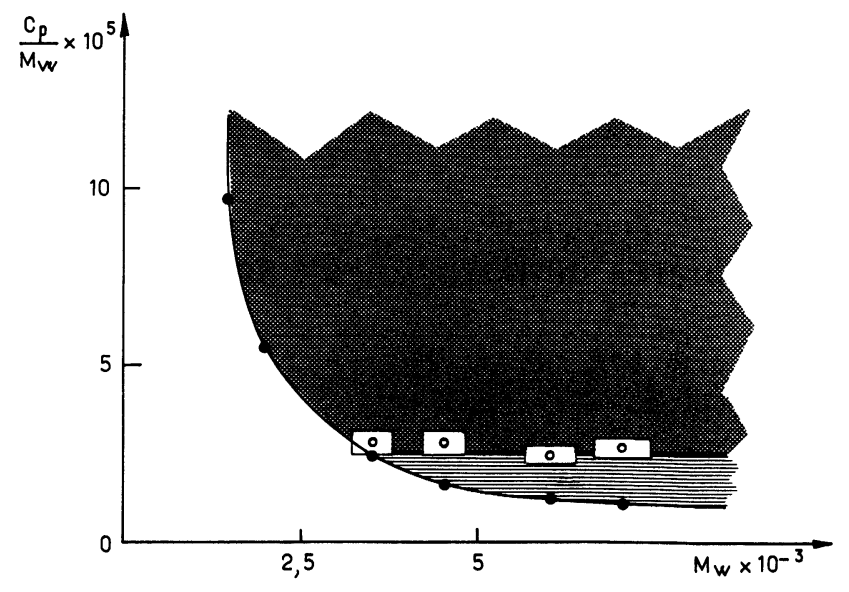

Fig. 2. - Seuil d'apparition de la texture à fils fins pour les solutions nématiques de Me-PNPM dans $\mathrm{H}_{2} \mathrm{SO}_{4}$ à $100 \%$. zone nématique à fils fins; : zone nématique à texture mal définie ; $\square$ : domaine d'erreur correspondant aux Mn calculées.

[Threshold conditions for the appearance of the threaded textures. : threaded texture of the nematic phase; texture of the nematic phase; $\square$ : uncertainty due to the calculation of $\mathrm{Mn}$.]

n'était toutefois pas possible, faute de pouvoir, soit mesurer directement $M_{\mathrm{n}}$ par les méthodes traditionnelles, soit calculer sa valeur avec une précision suffisante à partir de celles de $M_{\mathrm{w}}$ et de l'indice de polydispersité $M_{\mathrm{w}} / M_{\mathrm{n}}$ [2]. Remplacer $C_{\mathrm{v}}$ et $M_{\mathrm{n}}$ par $C_{\mathrm{p}}$ et $M_{\mathrm{w}}$ introduit une incertitude dont l'ampleur est illustrée par l'étendue des cadres qui entourent les points expérimentaux dans la figure 2 .

Il est difficile d'interpréter quantitativement l'observation que nous venons de faire, à savoir que la texture à fils fins apparaît au-delà d'une concentration molaire bien définie et correspondant à une densité d'extrémités de chaînes, indépendante de la masse moléculaire. Cependant, on ne peut s'empêcher de rapprocher cette observation des remarques que nous avons déjà faites [1], lorsque nous avons proposé le modèle de divergence pure radiale. Rappelons que nous avons alors mentionné (cf. annexe, réf. [1]) que la création d'une disinclinaison présentant ce type d'arrangement moléculaire implique, dans le cas de molécules de haut poids moléculaire, un processus de diffusion d'extrémités de chaînes vers le centre du défaut ; ce processus est d'autant plus rapide que la densité d'extrémités de chaînes dans le milieu est élevée, c'est-à-dire que la masse moléculaire est faible. Il est certain que cet argument supplémentaire ne peut être considéré comme preuve définitive du modèle proposé ; mais, faute d'observations directes au microscope démontrant le rang +1 des lignes de disinclinaison, il nous a semblé intéressant de l'exposer brièvement dans cette note.

\section{Bibliographie}

[1] Millaud, B., Thierry, A., Skoulios, A., J. Physique 39 (1978) 1109.
[2] Millaud, B., Strazielle, C., Polymer 20 (1979) 563.

[3] Du Pont de Nemours EI, BF no 2310426 (1976). 